\title{
Evaluation of the Heart Rate and Arrhythmias following the Maze Procedure for Chronic Atrial Fibrillation
}

\author{
Bartira Cunha, Renato A. K. Kalil, Álvaro S. Albrecht, Gustavo G. Lima, José Cláudio L. Kruse \\ Porto Alegre, RS - Brazil
}

\begin{abstract}
Purpose - To assess the presence and the prevalence of arrhythmias and the variability of the heart rate in the medium-term postoperative period following the maze procedure for chronic atrial fibrillation $(A F)$.
\end{abstract}

Methods - Seventeen patients with a mean age of $51.7 \pm 12.9$ years, who previously underwent the maze procedure without cryoablation for chronic atrial fibrillation, were evaluated with the 24 hour electrocardiogram (ECG) - Holter monitoring from the $6^{\text {th }}$ month after the operation. Valvular and coronary procedures were concomitantly performed.

Results - The mean heart rate during Holter monitoring was $82 \pm 8 \mathrm{bpm}$; the maximal heart rate was $126 \pm$ $23 \mathrm{bpm}$ and the minimal heart rate $57 \pm 7 \mathrm{bpm}$. Sinus rhythm was found in $10(59 \%)$ patients and atrial rhythm was found in $7(41 \%)$. Supraventricular extrasystoles had a rate of $2.3 \pm 5.5 \%$ of the total number of heartbeats and occurred in $16(94 \%)$ patients. Six (35\%) patients showed nonsustained atrial tachycardia. Ventricular extrasystoles, with a rate of $0.8 \pm 0.5 \%$ of the total heartbeats, occurred in $14(82 \%)$ patients. The chronotropic competence was normal in $9(53 \%)$ patients and attenuated in $8(47 \%)$. The atrioventricular conduction (AV) was unchanged in $13(76 \%)$ patients and there were 4 (24\%) cases of first degree atrioventricular block (AVB).

Conclusion-After the maze procedure, the values for the mean heart rate, AV conduction and chronotropic competence approach the normal range, although some cases show attenuation of the chronotropic response, first degree AV block or benign arrhythmias.

Key words: atrial fibrillation, surgery, arrhythmias

Instituto de Cardiologia do Rio Grande do Sul/Fundação Universitária de Cardiologia

Mailing address: Renato A. K. Kalil - Unidade de Pesquisa - Av. Princesa Isabel, 395 - 90620-001- Porto Alegre, RS - Brazil
Atrial fibrillation (AF), either alone or in combination with other heart diseases, is the most prevalent of all arrhythmias ${ }^{1}$. It is considered a clinically relevant condition because of its symptoms, the poor response to drug therapy and, above all, because of its potential of causing thromboembolic events ${ }^{2}$.

Aiming to totally correct this arrhythmia and remove its detrimental sequelae, Cox et $\mathrm{al}^{3}$, in 1987, developed the maze surgical technique. In this procedure-indicated for the treatment of primary or secondary chronic AF - multiple atrial incisions are made to create an electrical maze ${ }^{4}$.

In order to test the efficacy of this technique, some criteria related to the healing of AF must be met ${ }^{3}$; one of them is the recovery of the sinus rhythm (SR). However, the nature and the electrocardiographic profile of the heart rhythm after the maze procedure have not been totally studied yet. In this report, electrocardiographic monitoring on an outpatient basis was performed to screen arrhythmias, define the prevalence of these arrhythmias, and provide information on the variability of the heart rate in the medium-term postoperative period (PO).

\section{Methods}

From June 1995 to October 1997, 17 patients who previously underwent the maze procedure for chronic AF were evaluated using 24 hour electrocardiographic monitoring (Holter).

Patients who met the following inclusion criteria were eligible for surgery: presence of chronic AF, which was refractory for a period of 6 months or longer, in patients who had undergone the first heart surgery and/or who had shown a previous episode of stroke.

Sixteen $(94 \%)$ patients had secondary AF and underwent other surgical procedures concomitantly, including mitral valvoplasty, $13(82 \%)$ patients; mitral valvoplasty plus myocardial revascularization, $1(6 \%)$; prosthetic mitral valve, 1 (6\%); and aortic valvoplasty, 1 (6\%). Only one patient underwent the maze procedure alone. 
The valvular diseases were of rheumatic etiology in 13 $(82 \%)$ cases. Of these, mitral stenosis was the most prevalent, with $6(46 \%)$ cases, followed by mitral stenosis and regurgitation alone, with 4 (31\%), and mitral stenosis and regurgitation plus tricuspid regurgitation, and mitral stenosis and regurgitation plus aortic stenosis and regurgitation, $1(8 \%)$ case each. In addition, there was $1(8 \%)$ case of mitral regurgitation. Valvar abnormalities of nonrheumatic etiology were represented solely by mitral regurgitation, in $3(19 \%)$ patients. The single patient in whom the arrhythmia was the primary condition was a patient with refractory paroxysmal AF, who head an unsuccessful AV ablation .

The age of the patients ranged from 31 to 78 years (mean, 52 \pm 13 years). There were $12(70 \%)$ females and 5 $(30 \%)$ males. The PO time interval corresponding to the Holter evaluation ranged from 6 to 29 months (mean $=12.7 \pm$ 7.3). Nine (55\%) patients had previously used the following antiarrhythmic drugs: digoxin, 7 (77\%); amiodarone, 1 (11\%); and beta-blockers, 1 (11\%). During the preoperative evaluation, $1(6 \%)$ patient fulfilled the criteria for functional class (FC) II; $15(88 \%)$ belonged to FC III and $1(6 \%)$ to FC IV. During clinical follow-up on an outpatient basis, conducted concomitantly with the electrocardiographic monitoring, there were $16(94 \%)$ patients in FC I and $1(6 \%)$ patient in FC II. In the same period, the mean values for the diameter of the left atrium and the ejection fraction using the echocardiographic analysis were, respectively, 4.96 \pm 1.0 (range, 3.1 to $6.6 \mathrm{~cm}$ ) and 63.45 \pm 4.8 (range, 56 to $70.4 \%$ ).

SR was detected in fifteen ( $88 \%$ ) patients during the early $\mathrm{PO}$, and atrial rhythm and junctional rhythm were reported in one case each $(6 \%)$. In the early PO period, 8 (47\%) patients required temporary pacing due to atrial bradycardia or transient total atrioventricular block (AVB).

The surgical procedure performed was essentially the same procedure first described by Cox and subsequently modified. It is the method currently employed and named Cox $3{ }^{5}$. However, we did not use the cryoablation of the coronary sinus and of the intersections with the rings of the mitral and tricuspid valves. Instead of cryoablation, a more extensive dissection of the site, followed by electrocoagulation, was performed.

From the $6^{\text {th }}$ month after surgery, a medical history and physical examination was obtained from the patients in a specific outpatient clinic, and ambulatorial $24 \mathrm{~h}$ electrocardiographic monitoring was performed on these patients to detect the presence, the type, and the prevalence of arrhythmias. The electrocardiographic findings were correlated with the symptoms, and the chronotropic response during regular physical activities and the influence of the medication in the postoperative heart rate were also evaluated.

The rhythm was assessed and characterized during Holter monitoring based on the evaluation performed by independent observers of the morphology of $\mathrm{P}$ waves in V2 and V5 leads.

On the other hand, when the tracings of the 12-lead
ECG obtained at the same time as the Holter monitoring were examined, the rhythm was characterized according to the $\mathrm{P}$ wave axis in the frontal and horizontal planes and according to the $\mathrm{P}$ wave morphology in the 12 leads.

As abnormalities of $\mathrm{P}$ wave morphology are known to occur in the maze procedure, a $\mathrm{P}$ wave was considered sinus when its axis was situated between $30^{\circ}$ and ${ }^{+} 90^{\circ}$ in the frontal plane with a plus-minus morphology in the $\mathrm{V}_{1}$ lead and when it showed a positive initial part in $\mathrm{V}_{5}$ and $\mathrm{V}_{6}$ leads.

It is important to emphasize that the changes in $\mathrm{P}$ wave morphology, its flattening and its changes in the length are abnormalities that may sometimes hamper the analysis and definition of the rhythm as atrial or sinus rhythm. This is a common finding during the PO period of the maze procedure.

The stability of the rhythm was confirmed by the visual analysis of the complete monitoring.

In order to make an analysis of the arrhythmias, the atrial arrhythmias were divided into 3 groups: isolated supraventricular extrasystoles (SVES), nonsustained atrial tachycardia (lasting no more than $30 \mathrm{sec}$.) and sustained atrial tachycardia (lasting more than $30 \mathrm{sec}$.). In the latter, major arrhythmias, such as atrial fibrillation or flutter, were reported. The ventricular extrasystoles (VES) were further subdivided as follows: rare (up to $30 / \mathrm{h}$ ); frequent (more than $30 / \mathrm{h}$ ) and complex - this subgroup included polymorphic, bigeminal, and paired extrasystoles, as well as ventricular tachycardia.

The tests were recorded in 2 or 3-channel Space-Labs recorders and in DMI-Burdick recorders with a real-time channel. The reading was done in a DMI-Burdick analyzer.

All patients were advised to make no changes in their daily routine during the time reserved for Holter recording.

For statistical analysis, the numeric values were expressed as mean \pm standard deviation. The Student $t$ test was used for continuous variables and the chi-square test for categorical variables. An $\alpha$ of 0.05 was considered $\operatorname{critical}(\mathrm{p}<0.05)$.

\section{Results}

The mean heart rate during electrocardiographic monitoring was $82 \pm 9 \mathrm{bpm}$ (range, 67 to $103 \mathrm{bpm}$ ), reaching the maximal value of $126 \pm 23 \mathrm{bpm}$ (range, 88 to $185 \mathrm{bpm}$ ) and the minimal value of $57 \pm 7 \mathrm{bpm}$ (range, 44 to $71 \mathrm{bpm}$ ).

Of the 17 patients, SR was detected in $10(59 \%)$ and atrial rhythm in $7(41 \%)$. In the group of patients with atrial rhythm, a case of wandering pacemaker was reported.

The SVES had a rate corresponding to $2.3 \pm 5.5 \%$ of the total heartbeats and were seen in $16(94 \%)$ patients. There was a larger number of isolated atrial ectopies, 41,883 SVES, 32,881 of which, however, were reported in one patient. Six $(35 \%)$ patients showed nonsustained atrial tachycardia, and there were no cases of sustained atrial tachycardia, atrial flutter or AF.

Overall, the number of VESs was significantly smaller compared to atrial ectopic beats $-0.8 \pm 0.5 \%$ of the absolute number of heartbeats. In 3 patients (18\%), no signs of ven- 
tricular arrhythmias were noted. Within the 3 VES categories - rare, frequent and complex - the first and the last ones were equally prevalent, $11(65 \%)$ patients, whereas those categorized as frequently affected $3(18 \%)$ patients.

The chronotropic competence, as documented by Holter monitoring, was reported to be in the normal range in $9(53 \%)$ patients, i.e., the maximal heart rate achieved represented more than $85 \%$ of the maximal calculated rate. Eight $(47 \%)$ patients showed attenuated chronotropic response according to the interpretation of the test.

The atrioventricular conduction (AV) remained unchanged in $13(76 \%)$ patients, and there were $4(24 \%)$ cases of first degree AVB.

No pauses above 2 seconds were observed. This explains the absence of escape rhythm in the electrocardiographic tracings.

Seven $(41 \%)$ patients reported some kind of symptom during the monitoring interval, but, of these, the symptomatology did not correlate with significant electrocardiographic abnormalities in $4(57 \%)$. In one patient, the symptom reported correlated with sinus tachycardia, in a second patient there was an association with frequent and isolated SVES and in a third patient, there was an association with isolated VES with periods of nonsustained supraventricular tachycardia (SVT). The distribution of supraventricular arrhythmias according to their prevalence is shown in Table I.

\section{Discussion}

The correction of the irregular heart rhythm characteristic of AF is one of the healing criteria to be met by the maze procedure in order to assess the efficacy of this procedure $^{3}$. In this study, the assessment of heart rhythm by ECG-Holter in the medium- and long-term PO period following the surgical procedure documented the presence of SR in $59 \%$ of the patients and atrial rhythm in $41 \%$, with a total of $100 \%$ showing preserved atrioventricular synchronism. These results are in accordance with those found in the literature, such as the study by Cox et al ${ }^{3}$, which included a five-year clinical follow-up and highlighted the recovery of the SR in $60 \%$ of their patients. Another more

\begin{tabular}{|c|c|c|c|}
\hline \multicolumn{4}{|c|}{$\begin{array}{l}\text { Table I - Distribution of supraventricular and ventricular } \\
\text { arrhythmias according to their prevalence }(n=17)\end{array}$} \\
\hline & \multicolumn{3}{|c|}{ Arrhythmias } \\
\hline & Atrial & Ventricular & $\%$ \\
\hline Absent & 1 & 3 & \\
\hline Isolated SVES & 16 & & 94 \\
\hline NST & 6 & & 35 \\
\hline ST & - & & - \\
\hline Rare & & 11 & 65 \\
\hline Frequent & & 3 & 18 \\
\hline Complex & & 11 & 65 \\
\hline $\begin{array}{l}\text { SVES - suprave } \\
\text { ST- sustained t }\end{array}$ & $\begin{array}{l}\text { extrasy } \\
\text { a. }\end{array}$ & - nonsustain & dia; \\
\hline
\end{tabular}

recent study by Vogt et al ${ }^{6}$, which similarly assessed a series of 17 patients, showed the presence of stable SR during a period of 13.7 months after the operation.

The difficulty in distinguishing the sinus rhythm from the atrial rhythm is well known. This difficulty results from the maze procedure itself, which modifies the morphology, the length and the amplitude of the $\mathrm{P}$ wave. The most important factor to be noted from the results of the surgical procedure is unlikely to be the name of the rhythm, but, instead, the evidence of preserved AV sequence of activation, a statement that is further proved elsewhere in this report. With the use of Doppler echocardiography, the effectiveness of the atrial contraction and atrioventricular contractile sequence was demonstrated in these patients ${ }^{7}$.

In relation to the frequency and prevalence of arrhythmias, the mean number of SVESs was as expected and did not significantly differ from a previous report ${ }^{6}$, where the presence of SVT was witnessed in 10 patients - these figures are higher than those of our series. The absolute number of isolated premature atrial beats was significantly higher compared with the two other forms of arrhythmias detected, i.e., sustained and nonsustained atrial tachycardia. Isolated premature atrial beats were most prevalent (94\%). Nonsustained tachycardias reached $35.3 \%$ and sustained tachycardias, $0 \%$. Based on this qualitative difference in the nature of the arrhythmias, it can be noted that the most prevalent type is also the less complex, thus showing a more benign nature. Another point that deserves mention is that, although the number of isolated atrial extrasystoles was not modest - a fact that can be partially explained by the high frequency of atrial ectopies shown in one patient $(32,881)-$ a minority of patients, i.e., 7 (41\%), reported some form of symptomatology during the monitoring interval and, in this group, only 3 had reports showing a correlation between the symptoms reported and major electrocardiographic abnormalities. Attenuation of the symptoms commonly related to $\mathrm{AF}$ is extremely relevant, as the significant distress experienced by patients with this conditions is well known.

The VESs were significantly less frequent than the atrial extrasystoles. However, a similar trend to the occurrence of both the rare and complex types of extrasystoles was noted. The intermediate type (frequent) was less representative. Although the analysis of premature atrial beats is the one that carries the greatest interest in the postoperative evaluation of patients with $\mathrm{AF}$, the fact that the ventricular rhythm disturbances, although present, are attenuated, is promising.

The mean heart rate $-82 \pm 9 \mathrm{bpm}$ - significantly approached the heart rate of the general population and, together with the minimal and maximal values, was also in accordance with the values reported in other studies ${ }^{6}$.

Chronotropic competence, which is known to be reduced during the first month after the maze procedure, gradually returns to normal and, in the medium- and longterm follow-up, an adequate response is expected. However, it must be emphasized that the ECG-Holter monitoring in this report did not measure this variable during exercise 
testing, but instead, during regular physical activities of the patients. Thus, in our series, the chronotropic competence was shown to be normal in $9(53 \%)$ and attenuated in 8 (47\%) patients.

Recent reports on the electrocardiographic findings during the PO period of the maze procedure have demonstrated a trend towards evaluating the recovery of the sinoatrial node after the surgical procedure ${ }^{6,8,9}$. It is estimated that multiple atrial incisions result in partial denervation of sympathetic and parasympathetic systems of the heart ${ }^{9}$. The studies that use the analysis of the variability of the heart rate point out that, similarly to what occurs with the chronotropic response during exercise, the autonomic tonus, clearly absent in the first month of the PO period, gradually returns to normal within 6-12 months after the surgical procedure. As the mean PO period in this study was $12.7 \pm 7.38$ months, we consider that the patients of this series had already recovered the autonomic innervation, at least partially. The analysis of the variability of the R-R interval was not included in our results.

Cox et $\mathrm{al}^{3}$, in their study of a 5 -year PO period after the maze procedure, reported the recovery of AV synchronism in $98 \%$ of the patients of their series as from the $3^{\text {rd }}$ month of PO. All of the 17 patients who underwent ECG-Holter within
3-17 months of PO, followed-up by Vogt et al, had normal AV conduction $^{6}$. In the present study, the AV conduction remained unchanged in $76 \%$ of the cases, and there were 4 cases of first degree AVB.

In conclusion, the results of this study show that $\mathrm{SR}$ is effectively and safely restored in the medium-term PO period after the maze procedure. The evaluation of variables, such as mean, minimal and maximal HR, AV conduction and chronotropic competence, also demonstrated that the figures obtained, which were similar or equivalent to those established within physiological limits, confirm that this procedure is an effective option for the resolution of chronic AF. In spite of the slightly high frequency of atrial arrhythmias, less complex ectopic beats were most prevalent. This can be expected as a residual sequela of the surgical procedure and due to the preoperative status itself in those suffering from a chronic arrhythmia associated with heart disease caused by severe valvular lesions. However, there is still a limited number of studies in patients undergoing the maze procedure. Thus, we believe that longer follow-up periods would contribute to these results, ensuring the reproducibility of this technique and promoting its use in a larger number of patients, who could potentially benefit from this surgical technique.

\section{References}

1. Cox JL, Schuessler RB, D'Agostino Jr HJ, et al. The surgical treatment of atrial fibrillation. III Development of a definitive surgical procedure. J Thorac Cardiovasc Surg 1991; 101: 569-83.

2. Cox JL, Boineau JP, Schuessler RB, et al. Sucessfull surgical treatment of atrial fibrillation. Review and clinical update. JAMA 1991; 266: 1976-80.

3. Cox JL, Boineau JP, Schuessler RB, Kater KM, Lappas DG. Five-year experience with the maze procedure for atrial fibrillation. Ann Thorac Surg 1993; 56: 814-24.

4. Kalil RAK. Cirurgia das taquiarritmias supraventriculares. In: Gomes MF, Azeredo MAV, Frison LI, Vitola D, et al. Rotinas em Cardiologia. Porto Alegre: Artes Médicas, 1996; 30: 325.

5. Cox JL, Jaquiss RDB, Schuessler RB, Boioneau JP. Modification of the maze procedure for atrial flutter and atrial fibrillation. II Surgical technique of the maze III procedure. J Throrac Cardiovasc Sur 1995; 110: 485-95.
6. Vogt PR, La Rocca HPB, Candinas R, et al. Temporary loss of cardiac autonomic innervation after the maze procedure. European Journal of Cardio-Thoracic Surgery $1997 ; 12: 75-81$.

7. Hatem D, Kalil RAK, Albrecht AS, et al. Echocardiographic analysis of atrial function after Maze procedure associated to mitral valvuloplasty. J Am Coll Cardiol 1998; 31(suppl C): 391.

8. Fukushima K, Emori T, Shimizu W, et al - Delayed improvement of autonomic nervous abnormality after the Maze procedure: time and frequency domain analysis of heart rate variability using 24 hour Holter monitoring. Heart 1997; 778: 499-504.

9. Pasic M, Grauhan O, Musci M, et al. The Cox-Maze III procedure: parallel normalization of sinus node disfunction, improvement of atrial function and recovery of the cardiac nervous system. J Thorac Cardiovasc Surg (no prelo). 